\title{
Philosophiques
}

\section{Livres reçus}

Volume 18, numéro 2, automne 1991

URI : https://id.erudit.org/iderudit/027168ar

DOI : https://doi.org/10.7202/027168ar

Aller au sommaire du numéro

\section{Éditeur(s)}

Société de philosophie du Québec

ISSN

0316-2923 (imprimé)

1492-1391 (numérique)

Découvrir la revue

Citer ce document

(1991). Livres reçus. Philosophiques, 18(2), 199-202.

https://doi.org/10.7202/027168ar

Ce document est protégé par la loi sur le droit d'auteur. L'utilisation des services d'Érudit (y compris la reproduction) est assujettie à sa politique d'utilisation que vous pouvez consulter en ligne.

https://apropos.erudit.org/fr/usagers/politique-dutilisation/
Cet article est diffusé et préservé par Érudit.

Érudit est un consortium interuniversitaire sans but lucratif composé de l’Université de Montréal, l'Université Laval et l'Université du Québec à Montréal. Il a pour mission la promotion et la valorisation de la recherche. https://www.erudit.org/fr/ 


\section{LIVRES REÇUS}

ALFÉRI, Pierre (1991), Chercher une phrase, Paris, Christian Bourgois éditeur, Collection «Détroits », ISBN 2-267-01025-9, 77 pp.

ANDOUZE, Jean; MUSSET, Paul; PATY, Michel (éds) (1990), Les particules et l'univers. La rencontre de la physique des particules, de l'astrophysique et de la cosmologie, Paris, PUF, 396 pp.

AUBENQUE, J.; BRUN, J.; MILLET, L. (dirs.) (1990/juillet-septembre), Les études philosophiques, $\mathrm{n}^{\circ} 3$, Heidegger, Paris, PUF, ISBN 2-13-043043-0, ISSN 0014-2166, 432 pp.

AUBENQUE, J.; BRUN, J.; MILLET, L. (dirs.) (1990/avril-juin), Les études philosophiques, $\mathrm{n}^{\circ} 2$, Philosophie allemande, Paris, PUF, ISBN 2-13-043044-9, 288 pp.

BABIN, Ronald; LEBLANC-RAINVILLE, Simone (éds) (1990), La paix comme projet de justice, Université de Moncton, Moncton, ACFAS 74, Les cahiers scientifiques, ISBN 289245-102-7, 183 pp.

BOLLACHER, M.; HENRARD, R.; KLEVER, W. (éds) (1989), Studia Spinozana, Volume 5 (1989), Spinoza and Literature, Königshausen \& Neumann, Würburg, 475 pp.

BOSS, Gilbert; LEGAULT, G. (1989), Introduction aux techniques de la philosophie. Analyse de l'idée de justice, Zurich, Éditions du Grand Midi, 149 pp.

BOULAD-AYOUB, Josiane (1989), Contre nous de la tyrannie... - Des relations idéologiques entre Lumières et Révolution, Lasalle (Québec), Hurtubise HMH, coll. Brèches, ISBN 2-89045867-9, $370 \mathrm{pp}$.

DAVIS, Steven (éd.) (1991), Pragmatics - A Reader, New York, Oxford University Press, ISBN 0-19-505898-4, 595 pp. 
DERRIDA, Jacques (1990), Du droit à la philosophie, Paris, Galilée, 663 pp.

DESPLAND, Michel (1991), La tradition française en sciences religieuses. Pages d'histoire, Québec, Université Laval: Cahiers de recherche en sciences religieuses, vol. 10, ISBN 2-9800687-5-6, 176 pp.

DÍAZ, A.; ECHEVERRIA, J.; IBARRA, A. (eds.) (1990), Structures in Mathematical Theories - Reports of the San Sebastian International Symposium, September 25-29, 1990, Servicio Editorial Universidad del País Vasco, Argitarapen Zerbitzua Euskal Herriko Unibertsitatea, ISBN 84-7585-263-7, 492 pp.

DUNN, John (1991), La pensée politique de John Locke - Une présentation historique de la thèse exposée dans les "Deux traités du gouvernement ", Paris, Léviathan PUF, ISBN 213-043774-5, ISSN 0989-4462, 286 pp.

FICHTE/SCHELLING (1991), Correspondance (1794-1802), "Epiméthée », Paris, P.U.F.

GAGNON, Serge (1990), Plaisir d'amour et crainte de Dieu Sexualité et confession au Bas-Canada, Les Presses de l'Université de Laval, Sainte Foy, ISBN 2-7637-7249-8, 202 pp.

GHINS, Michel (1987), L'inertie et l'espace-temps absolu de Newton à Einstein - Une analyse philosophique, Bruxelles, Académie royale de Belgique, Palais des académies, ISBN 2-80310085-1, ISSN 0378-7893, 238 pp.

GOHIER, Christiane (dir.) (1990), La formation fondamentale. Tête bien faite ou tête bien pleine?, Les Éditions Logiques, Montréal, ISBN 2-89381-028-4, 398 pp.

GRAVEL, Pierre (1991), Politique, femmes, pouvoir - Éléments pour une théorie de l'otage - Essai sur le theâtre de Jean Racine, Montréal, VBL éditeur, ISBN 2-89005-404-7, 189 pp.

GRONDIN, Jean (1991), Emmanuel Kant avant/après, Paris, Criterion, 1991, ISBN 2-903702-48-9, 204 pp.

GRONDIN, Jean (1991), Einführung in die philosophische Hermeneutik, Darmstadt, Wissenschftliche Buchgesellschaft, ISBN 3534-07480-7, 249 pp.

HÖFFE, Otfried (1991), La justice politique - Fondement d'une philosophie critique de droit et de l'État, Paris, Léviathan PUF., ISBN-2-13-043489-4, ISSN 0989-4462, 397 pp. 
de KONINCK, Thomas; PLANTY-BONJOUR, Guy (éds) (1991), La question de Dieu selon Aristote et Hegel, Paris, PUF, ISBN 2-13-043532-7, 430 pp.

LABARRE, Albert (1990), Histoire du livre, Paris, PUF, Que saisje?, ISBN 2-13-043253-0, $127 \mathrm{pp}$.

LACHANCE, Francine (1990), La québécie, Zurich, GMB, Éditions du Grand-Midi, ISBN 2-88093-110-X, 207 pp.

LAMONDE, Yvan (1991), Territoires de la culture québécoise, SainteFoy, Presses de l'Université Laval, ISBN 2-7637-7254-4, 293 pp.

de LATTRE, Alain (1990), Bergson - Une ontologie de la perplexité, Paris, PUF, Philosophie d'aujourd'hui, ISBN 2-13-0428673, ISSN 0768-0805, $328 \mathrm{pp}$.

LEROUX, Georges (1990), Plotin. Traité sur la liberté et la volonté de l'Un (Ennéade VI, 8 (39)). Introduction, texte grec, traduction et commentaire. Paris, Librairie Philosophique J. Vrin, (Histoire des doctrines de l'Antiquité classique, volume 15), $449 \mathrm{pp}$.

LORIES, Danielle (1989), Expérience esthétique et ontologie de l'oeuvre, Académie royale de Belgique, Bruxelles, Palais des académies, Collection in-8 - 2e série, T. LXVIII - Fascicule 1, ISBN 2-8031-0074-6, 286 pp.

MACHEREY, Pierre (1990), À quoi pense la littérature? Pratiques théoriques, Paris, PUF, 253 pp.

MARION, Jean-Luc (1991), Dieu sans l'être, Paris, Quadrige PUF, ISBN 213043905 5, ISSN 0291-0489, 283 pp.

MARTIN, Robert M. (1991), The Philosopher's Dictionary, Peterborough, Broadview Press, ISBN 0-921149-75-1, 255 pp.

MATTÉI, Jean-François (1990), La naissance de la raison en Grèce, Actes du Congrès de Nice, Mai 1987, Paris, PUF, 437 pp.

PARRET, Herman (dir.) (1991), La communauté en paroles Communication, consensus, ruptures, Liège, Mardaga, Philosophie et langage, $265 \mathrm{pp}$.

POZZO, Riccardo (1989), Hegel: 'Introductio in philosophiam', Firenze, La Nuova Italia Editrice, ISBN 88-221-0706-3, 269 pp.

RASCHINI, Adélaïde Maria (dir.) (1989), Rosmini Pesatore Europeo - Atti del Congresso Internazionale, Roma, 26-29 ottobre 1988, Milano, Jaca Book, Edizioni Universitarie Jaca, ISBN 88-16-95064-1, 459 pp. 
RICOEUR, Paul, (1990), Soi-même comme un autre, Paris, Seuil, $425 \mathrm{pp}$.

RODIS-LEWIS, Geneviève (1990), L'anthropologie cartésienne, Paris, PUF Épiméthée, ISBN 2-13-043182-8, ISSN 0768-0708, 297 pp.

RUELLAND, Jacques G. (1991), De l'épistémologie à la politique - la philosophie de l'histoire de Karl R. Popper, Paris, PUF, Philosophie d'aujourd'hui, ISBN 2-13-043270-0, ISSN 07680805, 248 pp.

SOULEZ, Antonia (1991), La grammaire philosophique chez Platon, Paris, PUF, ISBN 2-13-043385-5, ISSN 0768-0805, 347 pp.

SOURIAU, Étienne (1990), Vocabulaire d'esthétique, Paris, PUF, $1408 \mathrm{pp}$.

THINÈS, George (1991), Existence et subjectivité. Études de psychologie phémoménologique, Bruxelles, Éditions de l'Université de Bruxelles, $249 \mathrm{pp}$.

TODOROV, Tzvetan (1991), Les morales de l'histoire, Paris, Grasset, 308 pp.

VACHER, Laurent-Michel (1990), L'empire du moderne - Actualité de la philosophie américaine, essai, Montréal, Les Herbes Rouges, ISBN 2-950051-60-1, 203 pp.

VANDERVEKEN, Daniel (1990), Meaning and Speech Acts - Volume 1, Principles of Language Use, Cambridge, Cambridge University Press, ISBN 0-521-37415-4, $244 \mathrm{pp}$.

VANDERVEKEN, Daniel (1991), Meaning and Speech Acts - Volume 2, Formal Semantics of Success and Satisfaction, Cambridge, Cambridge University Press, ISBN 0-521-38216-5, 196 pp.

VEILLARD-BARON, Jean-Louis (1991), Hegel, Paris, Que saisje?, PUF, ISBN-13-043801-6, 126 pp.

WILLIAMS, Bernard (1990), L'éthique et les limites de la philosophie, traduit de l'anglais par Anne-Marie Lescouret, Paris, Coll. NRF Essais, Gallimard, 276 pp. 